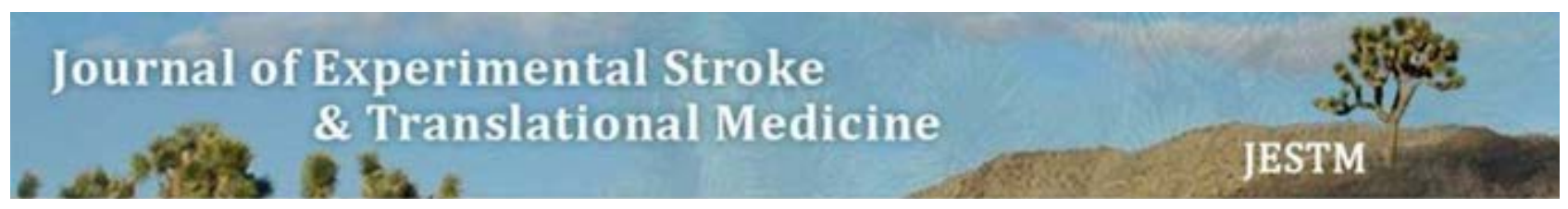

\title{
Machanism Associated with Apoptosis after Repetitive Transcranial Magnetic Stimulation in Permanent Stroke Rat Model
}

January 28, $2018 \cdot$ Volume $11 \cdot$ Original Research

Sheng Lan Jin ${ }^{1,2 \#}$, Min Kyun Sohn"\# and Sung Ju Jee ${ }^{1 *}$

${ }^{1}$ Department of Rehabilitation Medicine, School of Medicine, Chungnam National University, Daejeon, Korea

${ }^{2}$ Department of Rehabilitation, Yanbian University Hospital, Yanji, China

\#Contributed equally to this article

Article Citation: Sheng Lan Jin, Min Kyun Sohn, Sung Ju Jee. Machanism Associated with Apoptosis after Repetitive Transcranial Magnetic Stimulation in Permanent Stroke Rat Model. J Exp Stroke Transl Med. 2018 February. Online access at www.jestm.com

Correspondence should be sent to: Sung Ju Jee, Department of Rehabilitation Medicine, School of Medicine, Chungnam National University, Daejeon, Korea, Tel: 042-338-2423, Fax: 042-256-6056, E-mail: drjeesungju@daum.net

\section{Abstract}

Purpose: Neuromodulation therapy has been used as an adjunct treatment to promote motor recovery in stroke patients. The objectives of the present study were to determine the effect of repetitive transcranial magnetic stimulation (rTMS) on neurobehavioural recovery, evoked potential, and underlying biological mechanisms associated with neuronal cell death in rats after permanent middle cerebral artery occlusion.

Methods: Our study included 10 control and 40 Sprague-Dawley (SD) rats; of them, 30 were successfully subjected to the induction of permanent middle cerebral artery occlusion $(M C A O)$ stroke model ( $r T M S=15$, sham rTMS $=15)$. Ten-Hertz high-frequency rTMS was applied to the ipsilesional forepaw motor cortex for 2 weeks in the rTMS group. The somatosensory-evoked potential (SSEP) and motorevoked potential (MEP) were used to evaluate the electrophysiological changes. The behavioural function of the stroke rats was evaluated using the rotarod and Garcia tests. Stroke area was measured using a histological staining technique. Immunoblotting was used to explore the mechanisms of rTMS associated with neuronal apoptosis.

Results: 10 control and 20 MCAO rats $(N r T M S=10$; Nsham $=10)$ completed the experimental course. Regarding MEP amplitude, repeated-measured analysis of variance (RMANOVA) showed a significant TIME effect $[F(2,0.106)=11.241, P<0.001]$ and TIME $\times$ INTERVENTION interaction $[\mathrm{F}(2,0.057)=6.053, \mathrm{P}=0.005]$ on MEP amplitude, suggesting the beneficial effect of $\mathrm{rTMS}$ on motor cortical excitability. Regarding peak to peak SSEP amplitude, RMANOVA showed a significant TIME effect $[F(2,4.920)=3.4551, P=0.042]$. In terms of latency to fall, RMANOVA identified a significant TIME effect $[F(7,16640.335)=24.425, P<0.001]$ and INTERVENTION $\times$ TIME interaction $[F(7,2348.295)=3.447, P=0.002]$. In terms of distance to fall, RMANOVA revealed a TIME effect $[F(7,101.679)=26.515$, $P<0.001]$ and INTERVENTION $\times$ TIME interaction $[F(7,13.607)=3.548, P<0.002]$. In terms of Garcia score, RMANOVA revealed a significant effect of TIME [F $(7,69.282)=153.689, \mathrm{P}<0.05]$ and INTERVENTION $\times$ TIME interaction $[F(7,2.282)=5.063, P<0.05]$, suggesting a beneficial effect of rTMS over sham treatment. In the Kruskal-Wallis test of endoplasmic reticulum (ER) stress protein, rTMS also inhibited the protein levels of glucose-regulated protein 78 (GRP78), CATT/EBP homologous protein (CHOP), and p-eukaryotic initiation factor $2 \alpha(\mathrm{elF} 2 \alpha)$.

Conclusion: 10-Hertz rTMS leads to a rapid recovery in behavioural performance after permanent MCAO stroke model and maintains increased ipsilesional motor cortex activity 2 weeks after rTMS. The underlying beneficial effects of rTMS may be associated with ER stress protein in the neuronal cell death pathway.

Key Words: Stroke, Repetitive transcranial magnetic stimulation (rTMS), Neuroplasticity, Apoptosis, Endoplasmic reticulum (ER) stress Introduction

Neuromodulation therapy, which can change brain activity, has gained attention in the field of neurorehabilitation [Walsh, et al., 2006]. It can be combined with conventional neurorehabilitation therapy before, during, and after therapy. It can modulate brain activity before and after rehabilitation treatment and has been used with brain-stimulating drugs to augment their effects [Alonso-Alonso, et al., 2007]. Thus, neuromodulation therapy is a very useful tool in neurorehabilitation to augment neurorehabilitation therapeutic activity.

Repetitive transcranial magnetic stimulation (rTMS) is a representative non-invasive method that can change the excitability of the focal neural circuits and induce more precise functional reorganisation of the cortex than electrical stimulation [Sokhadze EM, et al., 2014]. When rTMS was first introduced, it was mainly used in clinical trials for depression and Parkinson's disease. However, rTMS can reportedly improve motor, language, and memory functions in stroke patients [Maeda F, et al., 2000; Martin PI, et al., 2000]. Kim et al. reported that high-frequency rTMS of the affected motor cortex can facilitate practice-dependent plasticity and improve motor learning performance in chronic stroke victims and insisted that motor recovery may be associated with increased cortical excitability [Kim YH, et al., 2006]. However, the underlying mechanisms of this phenomenon remain unclear.

Studies of the mechanism of the beneficial effects of rTMS have also been conducted by several researchers. Some experiments in the healthy rat brain demonstrated that rTMS can mediate neural plasticity by enhancing the expressions of neurotransmitters and neurotrophins such as glutamate, N-methyl-D-aspartate and brain-derived neurotrophic factor. [Yue, et al., 2009; Muller, et al., 2000; Kole, 
et al., 1999].

In other ways, the underlying neurochemical mechanism of the beneficial effect of high-frequency rTMS in a stroke rat model has been proposed that it has a neuroprotective effect via modulating the apoptotic process [Antonsson, 2001; Fujiki, et al., 2003; Yoon, et al, 2011]. The apoptotic process is known to be directly regulated by members of the Bcl-2 family, including Bax (pro-apoptosis) and Bcl-2 (anti-apoptosis) [Antonsson, 2001; Reed, 2006]. Yoon et al showed significant behavioural improvement after high-frequency rTMS in a temporary stroke rat model. They also reported that Bcl-2 protein levels increased after treatment compared to sham stimulation. However, they showed a significant increase in a single protein among candidate proteins that was insufficient to conclude the underlying mechanism of rTMS [Yoon et al., 2011]. Thus, preclinical studies to explore the therapeutic mechanism of rTMS therapy in the brain after stroke are insufficient. No study to our knowledge simultaneously explored the changes in neurobehavioural functions and electrophysiological parameters in a permanent stroke model. Because the majority of stroke patients are not candidates for immediate reperfusion therapy, it is vital that potential therapeutic tools are studied using a permanent occlusion animal model as well [Eady et al., 2013].

After stroke, neuronal cell death was induced by a decrease in oxygen and glucose, which in turn induces glutamate release at the presynaptic level. The high glutamate level and subsequent activation of glutamatergic postsynaptic receptors are the main components of the cascade of sequential molecular events that induce neuronal death [Choi \& Rothman, 1990]. In the neuronal cell death cascade, the endoplasmic reticulum (ER) plays a crucial role since the glutamatergic postsynaptic receptor induces the ER stress that produces cell death by excessive intracellular calcium accumulation. In this process, the ER is an important subcellular organelle that is responsible for intracellular calcium homeostasis, protein secretion, and lipid biosynthesis [Pizzo \& Pozzan, 2007; Ren et al., 2012]. Bcl-2 family proteins are well central regulators of cell life and death between the ER and mitochondria [Reed JC. 2006]. Thus, here we aimed to explore the extent of neurobehavioural recovery and neurophysiological change after rTMS and the underlying therapeutic mechanism associated with neuronal cell death in a permanent stroke rat model.

\section{Materials and Methods}

\section{Animal model}

Male Sprague-Dawley rats $(250-300 \mathrm{~g})$ were allowed free access to food and water. Animals were housed in a colony room under controlled temperature $\left(22^{\circ} \mathrm{C}\right)$ and a $12: 12$ light-dark cycle. All animal experiments were approved by the Institutional Animal Care and Use Committee of the preclinical research institute at Chungnam National University Hospital.

40 male Sprague-Dawley (SD) rats were anaesthetised with an intraperitoneal injection of a $1.0 \%$ ketamine cocktail (ketamine:xylazine = 2:1, $2.4 \mathrm{mg} / \mathrm{kg}$ Ketara, Yuhanyanghang, Seoul, South Korea; and Rompun, Bayer HealthCare LLC, Shawnee Mission, Kansas, USA) and subjected to permanent left middle cerebral artery occlusion (MCAO) with a modification of Longa's methods [Bazeley et al., 2008; Longa, Weinstein, Carlson, \& Cummins, 1989]. However, we did not open the internal carotid artery (ICA), a concept of a permanent MCAO model [Eady et al., 2013]. In brief, the left common carotid artery, external carotid artery, and ICA were carefully exposed. A 4-0 nylon silicon rubber-coated monofilament (0.35-mm coating diameter, 2-3-mm coating length; Doccol Corporation, Sharon, MA, USA) was inserted into the ICA and gently advanced to occlude the origin of the left middle cerebral artery. A heating pad was applied to maintain the rat's body temperature at $37 \pm 0.5^{\circ} \mathrm{C}$ during the surgery. The 10 control SD rats that did not undergo the MCAO procedure underwent only a skin incision after anaesthesia.

\section{Neuromodulation}

The rTMS was administered at the hot spot in left cerebral hemisphere. Since all rats underwent the left MCAO procedure, use of the left hemisphere was not appropriate for determining the hot spot. Thus, we measured the hot spot in the right hemisphere and assumed that hot spot in the left hemisphere was symmetrical to it on the opposite side. An animal figure-eight magnetic coil (Magstim Rapid2, Carmarthenshire, UK) was used to deliver the TMS. The hot spot was symmetrically opposite where the largest MEP amplitude can be acquired using the smallest stimulation intensity. Hot spot position was determined using the data from previous study. [Rotenberg et al., 2010]. The stimulation was performed with $10 \mathrm{~Hz}, 90 \%$ of the resting motor threshold (RMT), 2-s stimulation, 6.4-s rest, 800 stimulations per session, and 10 sessions at a regular time for 2 weeks. The total stimulation was done 8,000 times. The sham stimulation was $30 \mathrm{~cm}$ above from hot spot with opposite coil direction with the same protocol (Figure 1).

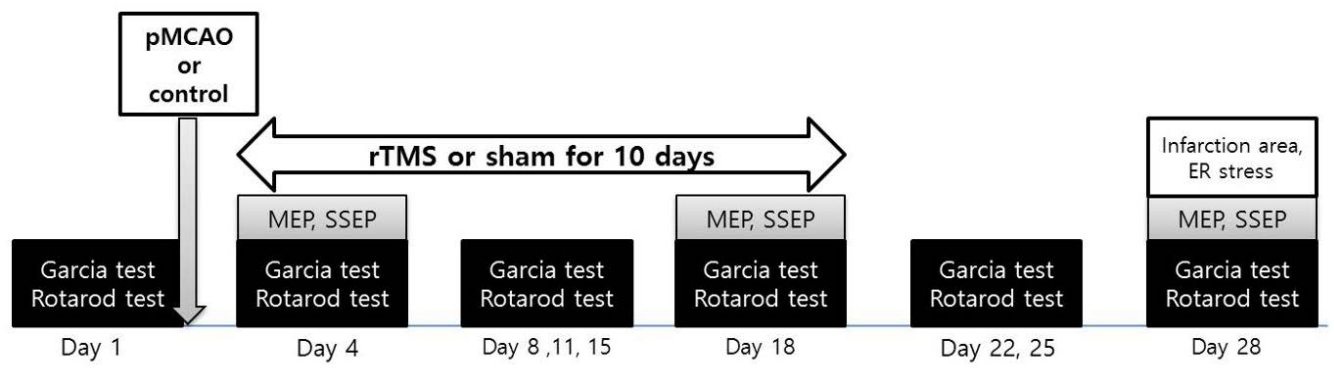

Figure 1.

Experimental design of neuromodulation study. Rats enrolled in rTMS or sham stimulation group in a random order. The rTMS (10 Hz, 2 $\mathrm{sec}$ stimulation with $6.4 \mathrm{sec}$ interval, total 800 stimulation/session) and sham (just sound) intervention were applied to the left motor cortex for 10 sessions with 5 consecutive and 2 resting days. Behavioral, electrophysiological tests (MEP, SSEP) were administered to assess the effects of intervention. At day 28 , infarction area and ER stress level were measured by TTC stain and immunoblotting, respectively. Abbreviation: pMCAO, permanent middle cerebral artery occlusion; rTMS, repetitive transcranial magnetic stimulation; MEP motor evoked potential; SSEP, somatosensory evoked potential; ER, endoplasmic reticulum.

\section{Electrophysiological evaluation}




\section{1) Motor evoked potential (MEP)}

After general anaesthesia with half a dose of the ketamine cocktail $(1.2 \mathrm{mg} / \mathrm{kg})$ was administered for the MCAO operation, the animals were fixed on the stereotaxic frame. The recording needle electrode was placed on the biceps muscle belly, the location of which was determined by palpation with the forepaw in the extended position. The reference needle electrode was positioned between the $2 \mathrm{nd}$ and 3 rd digit of the forepaw. The grounding electrode was inserted into the tail bed. The EMG signal was acquired with a setting according

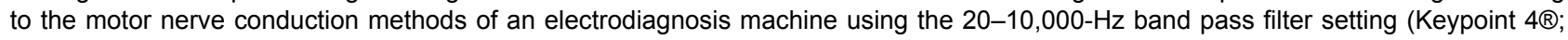
Dantec $^{\mathrm{TM}}$, Skovlunde, Denmark). At the hot spot, the stimulus intensity increased with $5 \%$ of machine output (MO) until MEP was observed in the unaffected forepaw. After the MEP was observed, the stimulus intensity was increased with $1 \%$ of MO to determine the RMT intensity. Based on the pilot biceps muscle TMS-derived MEP data in our laboratory, we defined RMT as the lowest stimulation intensity (\% MO) at which MEP can be acquired using a 0.015-mV amplitude five times with 10 consecutive stimulations [Luft AR, et al., 2001]. With these criteria, we identified a mean RMT of $70.3 \pm 3.7 \% \mathrm{MO}$ in the unaffected hemisphere at day 4 . The MEP was selected the signal with a stimulation intensity of $120 \%$ of the initial RMT on day 4 (Fig. 1). The MEP was obtained from the right biceps muscle at days 4 , 18 , and 28 . We used a maximal baseline value and the negative peak amplitude at each period was acquired for the statistical analysis.

\section{2) Somatosensory evoked potential (SSEP)}

SSEP was performed with the evoked potential and an electrodiagnosis system (Sierra Wave, Caldwell, Kennewick, WA, USA). The cathodal and anodal needle electrode was inserted in the biceps muscle bellies of the forepaw and palm, respectively. The electrical stimulator gave a $2.82-\mathrm{Hz} 0.1$-ms square-shaped wave. Stimulus intensity was twice that of the motor threshold, which was determined by visible muscle contraction [Bazley, et al., 2011; Agrawal, et al., 2010; Agrawal, et al., 2009; All et al., 2010].

The recording needle electrode was inserted $3.8 \mathrm{~mm}$ contralateral and $0.2 \mathrm{~mm}$ posterior to the bregma of the rat head according to a previous study [Agrawal et al., 2010]. The signal was amplified 10,000 times, filtered at a 10-500-Hz frequency, and averaged with 200 signals. Data acquired from the SSEP were latency to the first positive peak, latency to the first negative peak, and peak to peak amplitude between the first positive and negative peaks. The forepaw SSEP was obtained from the left hemisphere on days 4, 18, and 28 (Figure 2). To minimize capturing the side effects of anaesthesia, electrophysiological tests were not performed on day 1

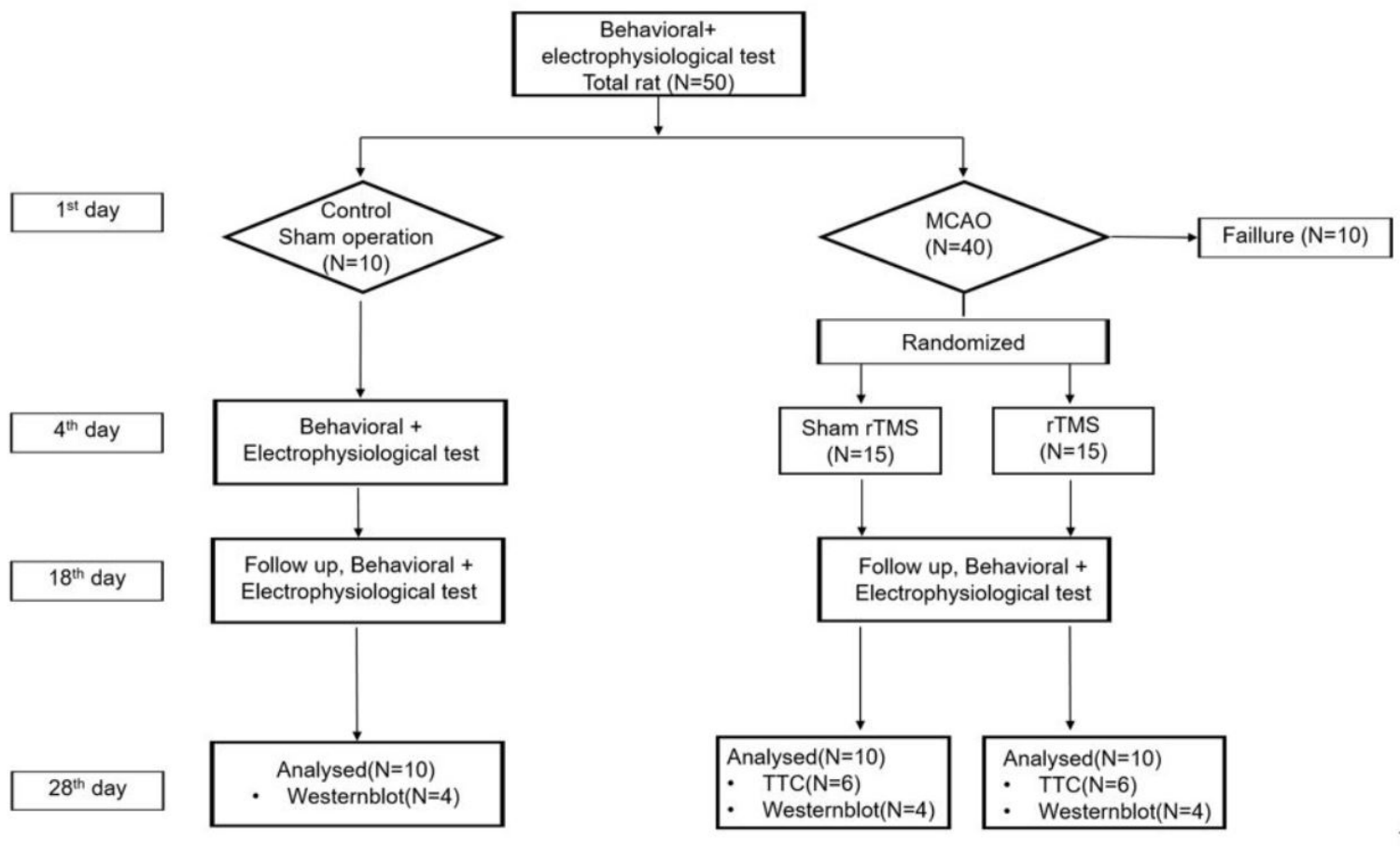

\section{Figure 2.}

Study flow and numbers of participated rats.

\section{Behavioral evaluation}

The behavioural tests, including the Garcia test and rotarod test, were performed on days 1 (before surgery), 4, 8, 11, 15, 18, 22, 25, and 28 (Figure 2). All animal experiments were conducted according to the National Institutes of Health Guide for the Care and Use of Laboratory Animals.

\section{1) Rota rod test}

The diameter of the rod was $9 \mathrm{~cm}$ and the rotation velocity was increased from five to 50 revolutions per minute (RPM) with a 5-RPM increase every 30 seconds. The performance was measured three times and averaged. The mean latency and distance to fall were recorded until that the rat fell from the rod or grasped the device. [Chen et al., 2001; Hamm et al., 1994].

\section{2) Garcia test}


The Garcia test includes six subtests: spontaneous activity, symmetry in the movement of the four limbs, forepaw outstretching, climbing, body proprioception, and response to vibrissae touch. The total score was 3-18, and a higher score indicates better performance [Garcia et al., 1995].

\section{Histologic and biochemical evaluation}

\section{1) Triphenyltetrazolium chloride staining}

To determine the infarct volumes, the rats were decapitated at day 28. Each brain was removed and sliced into coronal sections (2.0$\mathrm{mm}$ thick) using a rat brain slicer (Zivic Instruments, Pittsburgh, PA, USA). Five brain slices from each rat were incubated in $2 \%$ (w/v) triphenyltetrazolium chloride (TTC) solution for $20 \mathrm{~min}$ at $37^{\circ} \mathrm{C}$. Normal brain tissue was uniformly red in colour, whereas the infarcted regions were whitish. The brain slices from each rat were photographed and the extents of the whitish infarct areas were calculated using the ImageJ program (National Institutes of Health, Bethesda, MD, USA); the infarct volume was calculated as the percentage of the total brain area of each section.

\section{2) Immunoblotting}

The tissues in the peri-ischaemic zone were subjected to Western blotting. Protein was extracted through a serial procedure that involved the addition of protein extraction solution, homogenisation by ultrasonication for 15 seconds, and harvesting of the supernatant after centrifuging for $20 \mathrm{~min}$ at $12,000 \mathrm{rpm}$ at $4^{\circ} \mathrm{C}$. Protein quantification was performed using Breadford's method. Sodium dodecyl sulfatepolyacrylamide gel electrophoresis (SDS-PAGE) was administered and blotting was performed for 60 min at $110 \mathrm{~V}$, followed by 60 min at $60 \mathrm{~V}$. The SDS-PAGE gel was transferred onto nitrocellulose membrane at $90 \mathrm{~V}$ for 60 min. After the use of $5 \%$ bovine serum albumin (BSA) and $5 \%$ skim milk for $60 \mathrm{~min}$, the gels were probed with the appropriate primary antibodies overnight. The primary antibodies were mixed as GRP78 into 5\% BSA (1:2000), p-elF2 $\alpha$ into 5\% BSA (1:1500), CHOP into 5\% BSA, and $\beta$-actin into 5\% skim milk (1:2000). The nitrocellulose membrane was washed three times with Tris-buffered saline with Tween-20 (TBS-T) for 10 min each. Secondary antibodies were probed, with the appropriate 60 min blocking period. The secondary antibodies included polyclonal anti-rabbit and polyclonal antimouse mixed into 5\% BSA and 5\% skim milk (1:2000) each. In the final step, the nitrocellulose membrane was washed with TBS-T for 10 min and then TBS for $10 \mathrm{~min}$. The western blotting detection reagents were added to the nitrocellulose membrane and images were taken with an X-ray pre-processor. The present study utilised anti-GRP78, anti-CHOP, anti-p-elF2a (Cell Signaling Technology, Inc., Beverly, MA, USA), anti-elF2 $\alpha$ (Cell Signaling), and anti- $\beta$-actin (Santa Cruz, Biotechnology, Dallas, TX, USA) antibodies. Whole-cell lysates (30 $\mu \mathrm{g}$ ) were boiled in a SDS-PAGE gel-loading buffer, subjected to SDS-PAGE, transferred to a nitrocellulose membrane, and then probed with the appropriate primary and peroxidase-conjugated secondary antibodies (Santa Cruz). Chemiluminescent signalling was developed using Super Signal West Picoor Femto substrates (Pierce, Rockford, IL, USA), the blots were imaged with a Gel Doc 2000 ChemiDoc system (Bio-Rad, Hercules, CA, USA), and the band densities were quantified using Quantity One software (Bio-Rad). All values were normalised using $\beta$-actin as a loading control.

\section{Statistics}

SPSS (version 20.0; SPSS, Chicago, IL, USA) was used for the statistical analysis. Repeated-measures analysis of variance (RMANOVA) was used to explore the TIME effect and TIME $\times$ INTERVENTION interaction on the electrophysiological and behavioral data. If there was significant TIME effect and TIME $\times$ INTERVENTION interaction, a post-hoc analysis was performed using the Mann-Whitney $U$ test with Bonferroni's adjustment to determine the significant difference between groups at each evaluation time point. The infarction area between groups was compared using the Mann-Whitney $U$ test. Data from western blotting of the control, sham, and rTMS groups were compared using the Kruskal-Wallis test. If there was significant difference among group, post-hoc analysis was done to evaluate between group differences using the Mann-Whitney $U$ test with Bonferroni adjustment. Unless stated otherwise, $\mathrm{P}$ values < 0.05 were considered significant. All parameters are described as mean \pm standard deviation.

\section{Results}

30 rats for which the Garcia scores was between 7 and 13 at day 4 were randomly divided into the $r$ TMS $(n=15)$ and sham rTMS $(n=$ 15) groups [Lee et al., 2009]. In the end, $20(\mathrm{NrTMS}=10 ; \mathrm{Nsham}=10)$ and four control rats completed the entire experimental course. The data of 20 rats were subjected to the statistical analysis of behavioural change, electrophysiological change, and infarction area comparison by RMANOVA and an independent $t$ test. All data from 24 rats were analysed to compare proteins related with ER stress. At day 4, the latency to fall of the rotarod test of the rTMS and sham groups were $65.57 \pm 29.23$ and $67.66 \pm 29.22 \mathrm{sec}$, respectively (P > $0.05)$, while the distance to fall of the rotarod test was $2.21 \pm 1.64$ and $2.58 \pm 1.61 \mathrm{~m}$, respectively $(P>0.05)$. The Garcia test scores of the rTMS and sham groups at day 4 were $10.50 \pm 0.85$ and $10.20 \pm 0.92$, respectively. None of the parameters at day 4 were significantly different between the two groups on an independent $t$ test (Table 1).

\begin{tabular}{ccccc}
\hline & & & sham & \\
\hline & No of subjects & 10 & 1 \\
Rota rod test & 10 & $62.77 \pm 13.73$ & $68.54 \pm 12.14$ & 0.78 \\
& latency to fall $(\mathrm{s})$ & $2.06 \pm 0.80$ & $2.47 \pm 0.73$ & 0.76 \\
& distance to fall $(\mathrm{m})$ & $10.50 \pm 0.85$ & $10.20 \pm 0.92$ & 0.93 \\
\hline
\end{tabular}

Values are means \pm standard deviation. Abbreviations: $\mathrm{No}=$ number, $\mathrm{s}=\mathrm{sec}, \mathrm{m}=$ meters, $\mathrm{min}=$ minutes

Table 1.

Fundamental characteristics of permanent stroke rat model at day 4.

\section{Electrophysiological evaluation}

1) Motor evoked potential (MEP)

MEP amplitude showed a significant TIME effect $[F(2,0.106)=11.241, P<0.001]$ and TIME $\times$ INTERVENTION interaction $[F(2,0.057)$ $=6.053, P=0.005]$, suggesting the beneficial effect of rTMS on motor cortical excitability. The post-hoc Mann-Whitney $U$ test showed a 
tendency toward of larger amplitude in the rTMS group at day 18 and significantly larger amplitude in the rTMS group than the sham group at day 28. In terms of MEP latency, the data showed no significant TIME effect $[F(2,0.612)=1.311, P=0.282]$ of MEP latency. MEP data are shown in Table 2.

\begin{tabular}{|c|c|c|c|c|c|c|}
\hline & & Day 4 & Day 18 & Day 28 & TIME $^{\mathrm{b})}$ & TIME X INTERVENTIONc) \\
\hline & rTMS & $0.16 \pm 0.09$ & $0.25 \pm 0.13$ & $0.39 \pm 0.14$ & $0.002^{*}$ & $0.005^{\star *}$ \\
\hline \multirow[t]{3}{*}{ Amplitude (uV) } & Sham & $0.18 \pm 0.06$ & $0.17 \pm 0.08$ & $0.22 \pm 0.08$ & & \\
\hline & $P^{a)}$ & 0.353 & 0.029 & $0.011^{* \star}$ & & \\
\hline & rTMS & $2.77 \pm 1.03$ & $2.32 \pm 0.67$ & $2.23 \pm 0.67$ & 0.282 & 0.182 \\
\hline \multirow[t]{2}{*}{ Latency } & Sham & $2.40 \pm 0.46$ & $2.16 \pm 0.60$ & $2.16 \pm 0.60$ & & \\
\hline & $\mathrm{Pa})$ & 0.631 & 0.684 & 0.075 & & \\
\hline
\end{tabular}

Values are presented as mean \pm standard deviation. ${ }^{*} p<0.05,{ }^{* *} p<0.017$

a) $p$-values were derived from Mann Whitney $U$ test, b) p-values were derived from RMANOVA for the effect of TIME as the within subject factor (3 levels: 4, 18, and 28 days after stroke), c) p-values were derived from RMANOVA for the interaction between TIME and INTERVENTION (2 levels: rTMS and Sham).

\section{Table 2.}

Changes of amplitude and latency of MEP in affected forepaw at 4, 18 and 28 days after stroke.

\section{1) Somatosensory evoked potential (SSEP)}

In terms of peak to peak SSEP amplitude, analysis of variance (ANOVA) revealed a significant TIME effect $[F(2,4.920)=3.4551, P=$ $0.042]$ but no TIME $\times$ INTERVENTION interaction $[F(2,0.246)=0.173, P=0.842]$. No significant TIME effect was seen in terms of the latency of the first negative or first positive peak. Data of SSEP were shown in Table 3.

\begin{tabular}{ccccccc}
\hline & & Day $\mathbf{4}$ & Day $\mathbf{1 8}$ & Day 28 & TIME $^{\text {b) }}$ & TIME X INTERVENTIONc) \\
\hline $\begin{array}{c}\text { Peak to peak amplitude } \\
(\mathrm{uV})\end{array}$ & rTMS & $2.47 \pm 2.16$ & $2.86 \pm 2.03$ & $3.66 \pm 2.55$ & $0.042^{*}$ & 0.842 \\
& Sham & $2.26 \pm 1.91$ & $3.56 \pm 3.71$ & $3.02 \pm 2.45$ & & 0.658 \\
$\begin{array}{c}\text { Peak latency of } 1^{\text {st }} \\
\text { negative peak }\end{array}$ & rTMS & $11.06 \pm 1.77$ & $10.77 \pm 1.40$ & $10.72 \pm 1.82$ & $0.524^{\text {b) }}$ & 1.569 \\
& Sham & $10.25 \pm 2.29$ & $11.77 \pm 1.36$ & $11.46 \pm 1.84$ & $0.222^{\text {c) }}$ & 1.619 \\
$\begin{array}{c}\text { Peak latency of } 1^{\text {st }} \\
\text { positive peak }\end{array}$ & rTMS & $12.98 \pm 2.37$ & $12.90 \pm 2.25$ & $13.97 \pm 2.59$ & $0.212^{\text {b) }}$ & 1.392 \\
\hline
\end{tabular}

Values are presented as mean \pm standard deviation. ${ }^{*} p<0.05$.

${ }^{a)} p$-values were derived from Mann Whitney $U$ test, ${ }^{b} p$-values were derived from RMANOVA for the effect of TIME as the within subject factor ( 3 levels: 4,18 , and 28 days after stroke), ${ }^{c}$ p-values were derived from RMANOVA for the interaction between TIME and INTERVENTION.

Table 3.

Changes of amplitude and latency of SSEP in affected forepaw at 4, 18 and 28 days after stroke.

\section{Behavioral evaluation}

1) Rota rod test

Regarding fall latency, ANOVA revealed a significant TIME effect $[F(7,16640.335)=24.425, P<0.001]$ and INTERVENTION $\times$ TIME interaction $[F(7,2348.295)=3.447, P=0.002]$, suggesting a beneficial effect of $r T M S$ over sham on behavioural performance. The MannWhitney $\mathrm{U}$ test with Bonferroni adjustment showed that the latency to fall in the rTMS group was significantly higher than that of the sham group at days $15(P<0.001), 18(P<0.001), 25(P=0.002)$, and $28(P=0.003)$ but not at days 4, 8, 11, or 22 (Figure $3 A)$.

Regarding distance to fall, ANOVA revealed a significant TIME effect $[F(7,101.679)=26.515, P<0.001]$ and INTERVENTION $\times$ TIME interaction $[F(7,13.607)=3.548, P<0.002]$. The Mann-Whitney $\mathrm{U}$ test with Bonferroni adjustment showed that the distance to fall in the rTMS group was significantly farther than that of the sham group at days $15(P=0.002), 18(P<0.005), 25(P<0.004)$, and $28(P<0.001)$ but not at days 4, 8, 11, and 22 (Figure 3B). 
(A)



(B)

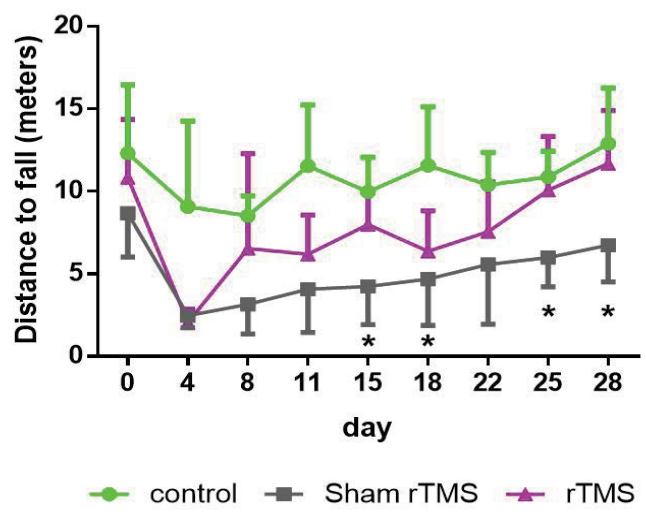

Figure 3.

Comparisons of latency $3(A)$ and distance $3(B)$ to fall from the rotarod before and after treatment. The rotaroad test showed significant TIME effect and TIME x INTERVENTION effect between rTMS and sham group by RMANOVA in both latency and distance. The Mann Whitney $\mathrm{U}$ test with Bonfferoni adjustment showed significantly higher latency and farther distance in rTMS compared to sham group at day $15,18,22,25$ and $28 .{ }^{*} p<0.006$, Mann Whitney $U$ test with Bonfferoni adjustment.

\section{2) Garcia test score}

Regarding Garcia test score, ANOVA revealed a significant TIME effect $[F(7,69.282)=153.689, P<0.05]$ and INTERVENTION $\times$ TIME interaction $[F(7,2.282)=5.063, P<0.05]$, suggesting a beneficial effect of $r T M S$ over sham. The Mann-Whitney $U$ test with Bonferroni adjustment showed that the mean Garcia test score in the rTMS group was significantly higher than that in the sham group at days $11(P$ $<0.001), 18(P<0.001), 22(P=0.002)$, and $25(P=0.003)$ but not at days $4,8,11$, and 28 (Figure 4).

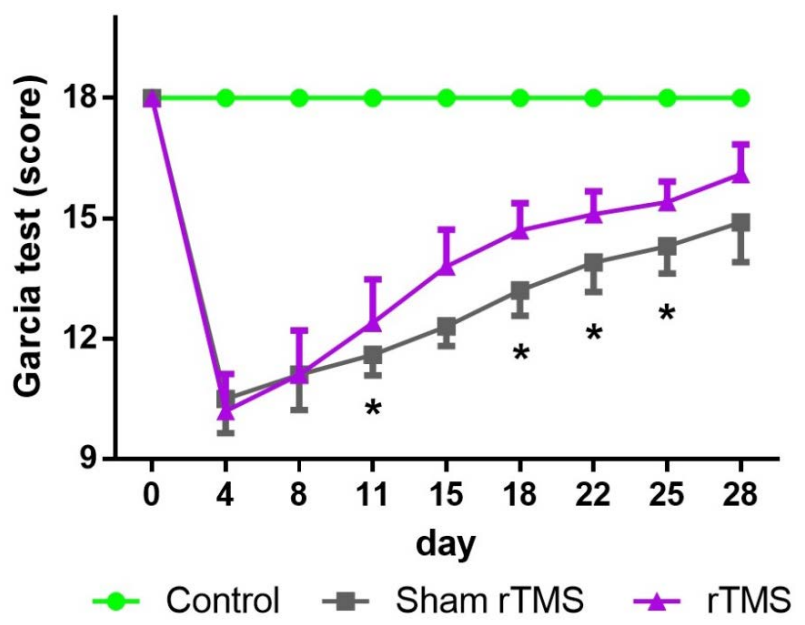

Figure 4. 
Comparison of score for Garcia test after treatment. Garcia score showed significant TIME effect and TIME X INTERVENTION interaction between rTMS and sham group by RMANOVA. The Mann Whitney $U$ test with Bonfferoni adjustment showed significant difference between groups at day 15, 18, 22 and 25. ${ }^{*} p<0.006$, Mann Whitney $U$ test with Bonfferoni adjustment.

\section{Histologic and biochemical evaluation}

\section{1) TTC stain for infarction area calculation}

TTC staining revealed that the major infarct area was the temporal and parietal lobes, striatum, and hippocampus of the left side of the rat brain. The infarction area in the rat brain at day 28 did not differ significantly between the rTMS and sham groups (Figure 5).

(A)

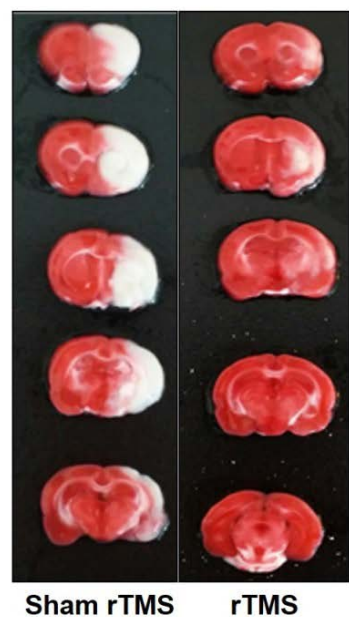

(B)

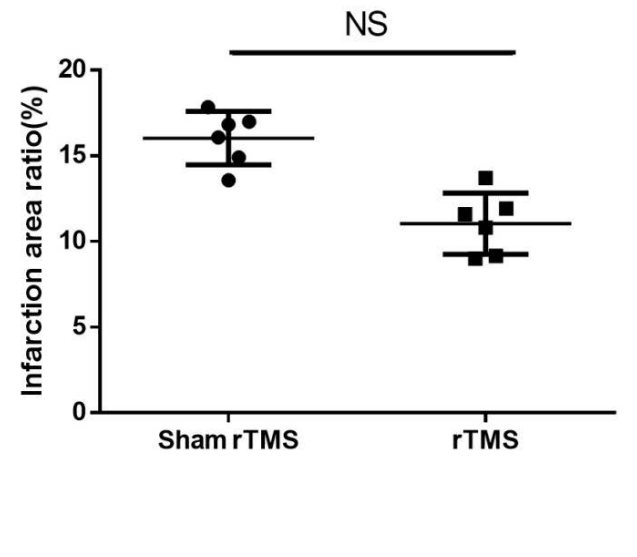

\section{Figure 5.}

The representative histological photograph with TTC stain for rat brain (A) and comparison of inarction area between groups (B). The infarction area of rTMS group didn't show significant difference compared to sham group.

\section{2) Immunoblotting}

On the Kruskal-Wallis test of the ER stress proteins, the levels of GRP 78, CHOP, and p-EIF2 $\alpha$ decreased significantly. In the post-hoc analysis, the Mann-Whitney $U$ test with Bonferroni adjustment showed significantly decreased amounts compared to the sham group in CHOP $(P=0.002)$ and GRP $78(P=0.022)$ but not $p$-elF2 $\alpha(P=0.097)$ (Figure 6$)$.
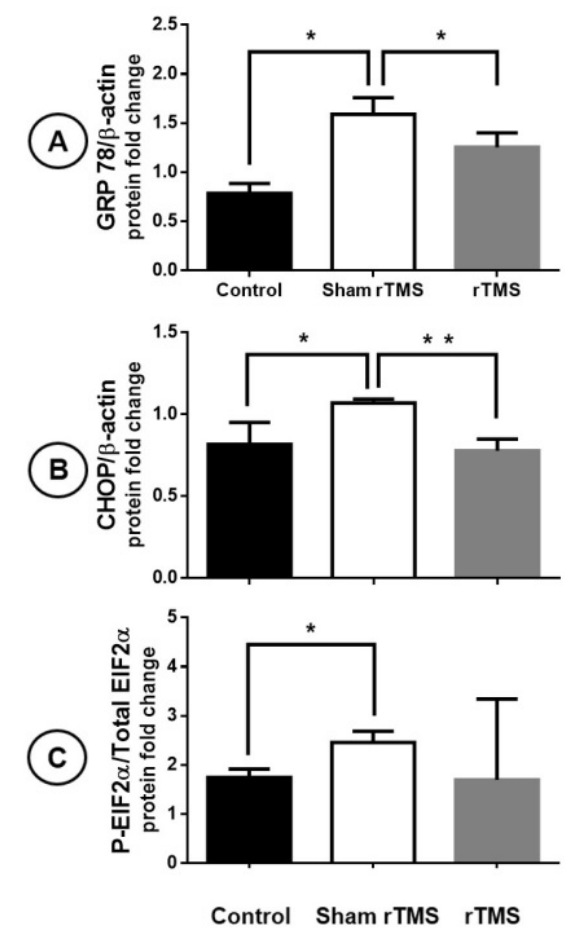
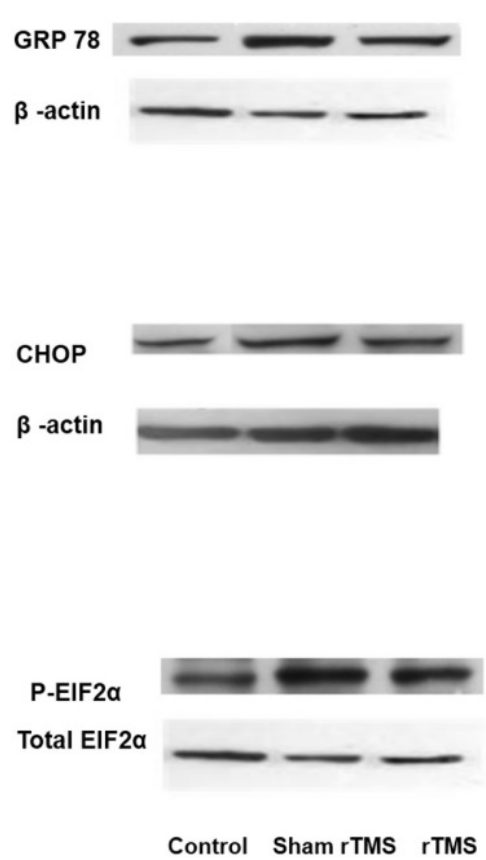

\section{Figure 6.}

The proteins such as GRP 78(A), CHOP (B) and p-eIF2 $\alpha(C)$ in ER stress pathways showed a tendency to increase amount in sham group 
compared to control. All data was presented as means standard deviation of three independent experiments ${ }^{*} p<0.05$, ${ }^{* *} p<0.01$. In rTMS group, the protein levels of GRP 78 and CHOP showed significant decrease compared to sham group, but p-elF2 $\alpha$ didn't show significant decrease of amount compared to sham group. All Western blots are representative of three independent experiments.

\section{Discussion}

This study aimed to explore the mechanism underlying the long-term beneficial effect of high-frequency rTMS on the ipsilateral hemisphere associated with behavioural recovery and electrophysiological change after stroke.

As our results demonstrated, the behavioural test and electrophysiological parameters showed a significant TIME effect and a significant TIME $\times$ INTERVENTION interaction after rTMS treatment except in SSEP. For the intergroup analysis at each time point, rats in the rTMS group showed significantly higher MEP and behavioural test performance. In the rTMS group, the GRP 78 and CHOP levels in the periinfarction area were significantly lower than those in the sham group, while the p-eIF2a protein levels associated with ER stress tended to be lower in the rTMS group than in the sham group. These data suggest that the underlying beneficial mechanism of rTMS can be associated with ER stress pathways. It is well known that ER stress can induce cell death and that various proteins are associated with the pathway, such as bcl-2 family, GRP 78, and p-elF2a. Our data elucidate the relationship between stroke-induced neuronal cell death and the beneficial effect of high-frequency rTMS.

Several studies have shown that ER stress is the main target role in ischaemia-induced neuronal deterioration. ER stress markers such as GRP78 and CHOP were reportedly upregulated and ischaemia-reperfusion injury induced neuronal apoptosis in the penumbra [Wu CX, et al., 2013]. On the other hand, GRP 78 can regulate CHOP expression, and its overexpression decreases $\mathrm{CHOP}$ induction, attenuating cell death [Fu HY, et al., 2008] or blocking ER stress [Rao RV., 2002]. A previous study on the association between oxidative stress and cardiovascular disease showed that antioxidants can be used to prevent cardiovascular disease [Street DA., et al., 1994; Bjelakovic G, et al., 2008; Myung SK, et al., 2013; Ciccone MM, et al., 2013]. Thus, our results support these findings.

Similarly, the present study found that permanent MCAO increased GRP78 and CHOP levels, which then decreased after rTMS treatment. Therefore, the neuroprotective effects of rTMS may be associated with inhibited CHOP expression and enhanced endogenous GRP78 activity in neuronal cells via inhibited microglia and astrocyte activation. One study reported that, in the brain injury induced by ER stress, CHOP induction is regulated by the PERK-elF2a-ATF4 pathway via an unfolded protein response [Zhang \& Kaufman, 2008]. In the present study, permanent MCAO instigated p-eIF2 $\alpha$ expression, which was inhibited by rTMS although not significantly. Therefore, the present findings suggest that rTMS can attenuate the p-elF2 $\alpha$ activation and lead to inhibited CHOP activity and cell apoptosis, as shown in a previous study [Kwon et al., 2015].

It is well known that the motor cortex has a strong modulatory effect on SSEP and MEP responses. For example, 1-Hz rTMS of the motor cortex at $110 \%$ of the active MT reduced SSEP amplitude without affecting latency [Enomoto et al., 2001]. In healthy subjects, a slow suprathreshold rTMS of the motor cortex significantly reduced N20-P25 SSEP amplitude [Coppola De Pasqua et al., 2012]. Coppola then showed that high-frequency rTMS failed to induce changes in SSEP amplitude compared to baseline in healthy subjects. In our study, SSEP did not change after rTMS in the ipsilesional motor cortex of the forepaw; this result can be explained by the fact that the neuroplastic effect of rTMS can be affected by neuronal damage. It also showed that the rTMS could significantly increase the peak MEP amplitude. Previous studies also confirmed that the change in MEP amplitude was related to brain cortex activity, which promoted brain reorganization [Concerto et al., 2018; Fitzgerald et al., 2006; Maeda et al., 2000].

Yoon [Yoon et al., 2011] reported a similar finding about the effect of high-frequency rTMS treatment in an MCAO model. The rTMS of 3,500 impulses with a $10-\mathrm{Hz}$ frequency also showed significant behavioural improvement such as the beam balance test and prehensile traction test in the treatment versus sham group. We used 8,000 impulses with a 10-Hz frequency and showed significant improvement in Garcia test and rotarod performance. Improvements within and between groups were significant at days 11 and 18 in the rTMS group. These behavioural changes are consistent with those of previous reports that high-frequency rTMS is beneficial to functional recovery after stroke [Loftus et al., 2014; Mosteller et al., 2014; Yoon et al., 2011]. However, we used a permanent stroke model, which features a larger infarction area and higher-severity impairments than a transient model. Thus, our results suggest that rTMS can be beneficial for more severe stroke patients as well.

Our study has some limitations. First, the sample size was small, leading to the use of non-parametric statistical methods and low statistical power. A larger sample size can result in more definite inter- and intragroup differences in dependent variables. Second, a future study with a larger sample size must explore various molecules associated with oxidative stress ER stress pathways and neuronal apoptosis. On the other hand, only electrophysiological evaluations were used to evaluate neuroplasticity in our study. Recent studies used imaging techniques like magnetic resonance imaging or electroencephalography to confirm changes in brain activity after rTMS.

\section{Conclusion}

In this study, 10- $\mathrm{Hz}$ rTMS led to a rapid recovery of behavioural performance in a permanent MCAO stroke model and maintained increased activity of the ipsilesional motor cortex about 2 weeks after rTMS. The underlying beneficial effects of rTMS may be associated with GRP 78 and CHOP protein expressions in the neuronal cell death pathway, whereas no significant changes were seen in other proteins like p-eIF2 $\alpha$. We must identify other molecules that increase GRP 78 expression or decrease CHOP expression. The present results may function as fundamental data regarding the basic mechanism of recovery after stroke.

\section{Funding}

This study was supported by grants (nos. HI10C2020, HI13C1990) from the Korean Health Technology R\&D Project and Ministry of Health \& Welfare (nos. NRF-2015R1C1A1A01055923, NRF-2017R1A2B4006500).

\section{References}

Agrawal G, Kerr C, Thakor NV, All AH (2010) Characterization of graded multicenter animal spinal cord injury study contusion spinal cord injury using somatosensory-evoked potentials. Spine (Phila Pa 1976) 35(11): 1122-27

Agrawal G, Thakor NV, All AH (2009) Evoked potential versus behavior to detect minor insult to the spinal cord in a rat model. J Clin Neurosci 16(8): 1052-55. 
All AH, Agrawal G, Walczak P, Maybhate A, Bulte JW, et al. (2010) Evoked potential and behavioral outcomes for experimental autoimmune encephalomyelitis in Lewis rats. Neurol Sci 31(5): 595-601.

Alonso-Alonso M, Fregni F, Pascual-Leone A (2007) Brain stimulation in poststroke rehabilitation. Cerebrovasc Dis 24(Supp1): 157-66.

Antonsson B (2001) Bax and other pro-apoptotic Bcl-2 family "killer-proteins" and their victim the mitochondrion. Cell Tissue Res 306(3): 347-61.

Bazeley PS, Shepelev V, Talebizadeh Z, Butler MG, Fedorova L, et al. (2008) snoTARGET shows that human orphan snoRNA targets locate close to alternative splice junctions. Gene 408(1-2): 172-79

Bazley FA, All AH, Thakor NV, Maybhate A (2011) Plasticity associated changes in cortical somatosensory evoked potentials following spinal cord injury in rats. Conf Proc IEEE Eng Med Biol Soc 2011: 2005-08.

Bjelakovic G, Nikolova D, Gluud LL, Simonetti RG, Gluud C (2008) Antioxidant supplements for prevention of mortality in healthy participants and patients with various diseases. Cochrane Database Syst Rev 16(2): CD007176

Chen J, Li Y, Wang L, Zhang Z, Lu D, et al. (2001) Therapeutic benefit of intravenous administration of bone marrow stromal cells after cerebral ischemia in rats. Stroke 32(4): 1005-11.

Choi DW, Rothman SM (1990) The role of glutamate neurotoxicity in hypoxic-ischemic neuronal death. Annu Rev Neurosci 13(1) $171-82$.

Ciccone MM, Cortese F, Gesualdo M, Carbonara S, Zito A, et al. (2013) Dietary intake of carotenoids and their antioxidant and antiinflammatory effects in cardiovascular care. Mediators Inflamm: 782137.

Concerto C, Boo H, Hu C, Sandilya P, Krish A, et al. (2018) Hypericum perforatum extract modulates cortical plasticity in humans. Psychopharmacology (Berl) 235(1): 145-53.

Coppola G, De Pasqua V, Pierelli F, Schoenen J (2012) Effects of repetitive transcranial magnetic stimulation on somatosensory evoked potentials and high frequency oscillations in migraine. Cephalalgia 32(9): 700-09.

Eady TN, Khoutorova L, Anzola DV, Hong SH, Obenaus A, et al. (2013) Acute treatment with docosahexaenoic acid complexed to albumin reduces injury after a permanent focal cerebral ischemia in rats. PLoS One 8(10): e77237.

Enomoto H, Ugawa Y, Hanajima R, Yuasa K, Mochizuki H, et al. (2001) Decreased sensory cortical excitability after 1 Hz rTMS over the ipsilateral primary motor cortex. Clin Neurophysiol 112(11): 2154-58.

Fitzgerald PB, Fountain S, Daskalakis ZJ (2006) A comprehensive review of the effects of rTMS on motor cortical excitability and inhibition. Clin Neurophysiol 117(12): 2584-96.

Fu HY, Minamino T, Tsukamoto O, Sawada T, Asai M, et al. (2008) Overexpression of endoplasmic reticulum-resident chaperone attenuates cardiomyocyte death induced by proteasome inhibition. Cardiovasc Res 79(4): 600-10.

Fujiki M, Kobayashi H, Abe T, Kamida T (2003) Repetitive transcranial magnetic stimulation for protection against delayed neuronal death induced by transient ischemia. J Neurosurg 99(6): 1063-69.

Garcia JH, Wagner S, Liu KF, Hu XJ (1995) Neurological deficit and extent of neuronal necrosis attributable to middle cerebral artery occlusion in rats. Statistical validation. Stroke 26(4): 627-35

Hamm RJ, Pike BR, O'Dell DM, Lyeth BG, Jenkins LW (1994) The rotarod test: an evaluation of its effectiveness in assessing motor deficits following traumatic brain injury. J Neurotrauma 11(2): 187-96.

Kim YH, You SH, Ko MH, Park JW, Lee KH, et al. (2006) Repetitive transcranial magnetic stimulation-induced corticomotor excitability and associated motor skill acquisition in chronic stroke. Stroke 37(6): 1471-76.

Kole MH, Fuchs E, Ziemann U, Paulus W, Ebert U (1999) Changes in 5-HT1A and NMDA binding sites by a single rapid transcranial magnetic stimulation procedure in rats. Brain Res 826(2): 309-12.

Kwon SK, Ahn M, Song HJ, Kang SK, Jung SB, et al. (2015) Nafamostat mesilate attenuates transient focal ischemia/reperfusion-induced brain injury via the inhibition of endoplasmic reticulum stress. Brain Res 1627: 12-20.

Lee SU, Kim DY, Park SH, Choi DH, Park HW, et al. (2009) Mild to moderate early exercise promotes recovery from cerebral ischemia in rats. Can J Neurol Sci 36(4): 443-49

Loftus JP, Cavatorta D, Bushey JJ., Levine CB, Sevier CS, et al. (2014) The 5-lipoxygenase inhibitor tepoxalin induces oxidative damage and altered PTEN status prior to apoptosis in canine osteosarcoma cell lines. Vet Comp Oncol. 14(2): e17-30.

Longa EZ, Weinstein PR, Carlson S, Cummins R (1989) Reversible middle cerebral artery occlusion without craniectomy in rats. Stroke 20(1): 84-91.

Luft AR, Kaelin-Lang A, Hauser TK, Cohen LG, Thakor NV, et al. (2001) Transcranial magnetic stimulation in the rat. Exp Brain Res 140(1): 112-21.

Maeda F, Keenan JP, Tormos JM, Topka H, Pascual-Leone A (2000) Interindividual variability of the modulatory effects of repetitive transcranial magnetic stimulation on cortical excitability. Exp Brain Res 133(4): 425-30.

Martin PI, Naeser MA, Theoret H, Tormos JM, Nicholas M, et al. (2004) Transcranial magnetic stimulation as a complementary treatment for aphasia. Semin Speech Lang 25(2): 181-91.

Mosteller M, Condreay LD, Harris EC, Ambery C, Beerahee M, et al. (2014) Exploring the roles of UGT1A1 and UGT1A3 in oral clearance of GSK2190915, a 5-lipoxygenase-activating protein inhibitor. Pharmacogenet Genomics 24(12): 618-21. 
Muller MB, Toschi N, Kresse AE, Post A, Keck ME (2000) Long-term repetitive transcranial magnetic stimulation increases the expression of brain-derived neurotrophic factor and cholecystokinin mRNA, but not neuropeptide tyrosine mRNA in specific areas of rat brain. Neuropsychopharmacology 23(2): 205-15.

Myung SK, Ju W, Cho B, Oh SW, Park SM, et al. (2013) Efficacy of vitamin and antioxidant supplements in prevention of cardiovascular disease: systematic review and meta-analysis of randomised controlled trials. BMJ 346: f10.

Pizzo P, Pozzan T (2007) Mitochondria-endoplasmic reticulum choreography: structure and signaling dynamics. Trends Cell Biol 17(10): 511-17.

Rao RV, Peel A, Logvinova A, del Rio G, Hermel E, et al. (2002) Coupling endoplasmic reticulum stress to the cell death program: role of the ER chaperone GRP78. FEBS Lett 514(2-3): 122-28.

Reed JC (2006) Proapoptotic multidomain Bcl-2/Bax-family proteins: mechanisms, physiological roles, and therapeutic opportunities. Cell Death Differ 13(8): 1378-86.

Ren G, Baritaki S, Marathe H, Feng J, Park S, et al. (2012) Polycomb protein EZH2 regulates tumor invasion via the transcriptional repression of the metastasis suppressor RKIP in breast and prostate cancer. Cancer Res 72(12): $3091-04$.

Rotenberg A, Muller PA, Vahabzadeh-Hagh AM, Navarro X, Lopez-Vales R, et al. (2010) Lateralization of forelimb motor evoked potentials by transcranial magnetic stimulation in rats. Clin Neurophysiol 121(1): 104-08.

Sokhadze EM, El-Baz AS, Sears LL, Opris I, Casanova, MF (2014) rTMS neuromodulation improves electrocortical functional measures of information processing and behavioral responses in autism. Front Syst Neurosci 8: 134

Street DA, Comstock GW, Salkeld RM, Schuep W, Klag MJ (1994) Serum antioxidants and myocardial infarction. Are low levels of carotenoids and alpha-tocopherol risk factors for myocardial infarction? Circulation 90(3): 1154-61.

Walsh V, Desmond JE, Pascual-Leone A (2006) Manipulating brains. Behav Neurol 17(3-4): 131-34.

Wu CX, Liu R, Gao M, Zhao G, Wu S, et al. (2013) Pinocembrin protects brain against ischemia/reperfusion injury by attenuating endoplasmic reticulum stress induced apoptosis. Neurosci Lett 546: 57-62.

Yoon KJ, Lee YT, Han TR (2011) Mechanism of functional recovery after repetitive transcranial magnetic stimulation (rTMS) in the subacute cerebral ischemic rat model: neural plasticity or anti-apoptosis? Exp Brain Res 214(4): 549-56.

Yue L, Xiao-lin H, Tao S (2009) The effects of chronic repetitive transcranial magnetic stimulation on glutamate and gamma-aminobutyric acid in rat brain. Brain Res 1260: 94-9.

Zhang K, Kaufman RJ (2008) From endoplasmic-reticulum stress to the inflammatory response. Nature 454(7203): 455-62. 